\title{
THEORETICAL MODEL OF ACOUSTIC WAVE PROPAGATION IN SHALLOW WATER
}

\author{
Eugeniusz Kozaczka \\ Grażyna Grelowska \\ Gdańsk University of Technology, Poland
}

\begin{abstract}
TThe work is devoted to the propagation of low frequency waves in a shallow sea. As a source of acoustic waves, underwater disturbances generated by ships were adopted. A specific feature of the propagation of acoustic waves in shallow water is the proximity of boundaries of the limiting media characterised by different impedance properties, which affects the acoustic field coming from a source situated in the water layer "deformed" by different phenomena. The acoustic field distribution in the real shallow sea is affected not only by multiple reflections, but also by stochastic changes in the free surface shape, and statistical changes in the seabed shape and impedance. The paper discusses fundamental problems of modal sound propagation in the water layer over different types of bottom sediments. The basic task in this case was to determine the acoustic pressure level as a function of distance and depth. The results of the conducted investigation can be useful in indirect determination of the type of bottom.
\end{abstract}

Keywords: shallow water acoustics, propagation of acoustic waves in water layer

\section{INTRODUCTION}

Propagation of acoustic waves in a shallow sea differs fundamentally from the same phenomenon occurring in the deep sea; due to the non-negligible distance from the sea bottom in the former case, where the presence of two regions limiting the water layer is the reason why the acoustic pressure distribution induced by a harmonic source has the interferential nature resulting from multi-path propagation of the acoustic signal ${ }^{1}$. These interferential properties of the acoustic field in the water layer limited by the free surface from one side and the bottom from the other, manifest themselves by forming a series of wave modes ${ }^{2}$. For this reason, we deal with the group velocity when discussing the propagation of wave modes. The influence of the sea surface has the form of acoustic signal amplitude and phase fluctuations, while the bottom surface absorbs a portion of the acoustic energy and transfers it to the limiting medium. The scale of the scattering effect occurring on the free surface, and also on the sea bottom, depends on the ratio of the wavelength to the characteristic size of irregularities (the Rayleigh criterion).

The definition of "shallow water" was adopted in accordance with the criterion $\mathrm{h}<10 \lambda$, which is generally accepted in the literature. Nevertheless, other criteria are also proposed in some papers. For instance, W.A. Kuperman and J.F. Lynch ${ }^{1}$ give: "In shallow water, with boundaries framed by the surface and bottom, the typical depth-to-wavelength ratio is about 10-100". Whereas L. Tolstoy and C.S. Clay ${ }^{5}$ introduce the distinction between deep and shallow sea at the point where the product of the horizontal wave number $\mathrm{k}_{\mathrm{r}}$ and the water depth $\mathrm{h}$ is such that $\mathrm{k} h \leq 10$. As can be seen from the characteristics, for audible frequencies the sea will be considered shallow when its depth is of the order of thousands of meters, compared to centimetres for ultrasonic frequencies.

A specific feature of the propagation of acoustic disturbances in a shallow sea is the proximity of boundaries of the limiting media characterized by different impedance 
properties, which affects the acoustic field. Such environments are called the dispersion media, with dispersion of the second kind occurring mainly in this case $\mathrm{e}^{5,6}$.

As the boundary surfaces of the involved limiting media are most frequently defined not deterministically but rather stochastically (random bottom surface, stochastic free liquid surface), finding an analytical solution to the acoustic disturbance propagation problem formulated this way is extremely difficult. The theory of stochastic equations is relatively undeveloped, despite some progress in the related methods observed in recent years.

Further in the paper, fundamental problems of sound propagation are discussed for selected models of shallow sea. The noise generated by ships that contains low frequency components will be used as the source of underwater acoustic disturbances. A sample spectrum of the ship generated underwater noise is shown in Fig. 1.

The basic task in this case was to determine the acoustic pressure level as a function of distance and depth. Scattering phenomena were not considered in detail, as the adopted frequencies of acoustic disturbances allowed them to be neglected.

\section{NOISE IN SEA ENVIRONMENT}

The anthropogenic noise covers mainly low and mid frequency bands and often fulfils the criterion of shallow sea propagation ${ }^{4}$. The main source of the underwater noise is the commonly understood maritime transport ${ }^{7,8}$. There are fairways where shipping traffic is very intense, like on highways. The low and medium frequency noise propagates in the sea over long distances from its sources, with much lower natural attenuation than in the air. Moreover, the structure of the water environment creates a kind of waveguide with conducing conditions for noise propagation over long distances.

Traditional use of maritime areas for sailing and fishing has been extended nowadays to cover new types of technical activity. Large-scale constructions are appearing in the sea and at its vicinity, including: oil and gas rigs, gas and oil pipeline networks, electric energy transmission networks, road traffic routes (bridges, tunnels, artificial islands), communication lines (optical fibres) and installations for production of electrical energy.

But still the most important source of underwater noise is shipping. Using the classic classification, the noise generated by ships can be segregated into ${ }^{9,10,11}$ :

- noise generated by dynamically active devices situated inside and on the surface of the hull, mainly by propulsion and auxiliary engines, and the system of transport of mechanical energy - shafting,

- noise produced by ship propellers,

- acoustic effects connected with cavitation of propellers and flow around the underwater part of the hull.

At low ship speed, the ship's service generator is the main source of the underwater noise generated by the ship. It emits tonal components that contribute almost entirely to the total radiated noise power of the ship and are independent of ship speed. A few of them are strong enough to be contributors to a high-speed signature. The tonal levels of the ship's service diesel generator are nearly stable in amplitude and frequency ${ }^{10}$. The wide-band energy of the noise generated by the ship's service generator is proportional to the square of the generated power ${ }^{2}$.

At higher ship speed, discrete components appear in the spectrum of the underwater noise as a result of mechanical activity of propulsion engines and propellers. These components are mainly observed in the frequency range up to $100 \mathrm{~Hz}^{10}$.

The underwater noise spectrum contains components whose origins can be directly linked to the activity of ship mechanisms ${ }^{13}$. Sample spectra of the underwater noise produced by a cargo ship are shown in Fig. 1 and Fig. 2 in the form of narrow-band spectrum and $1 / 3$ octave band spectrum.

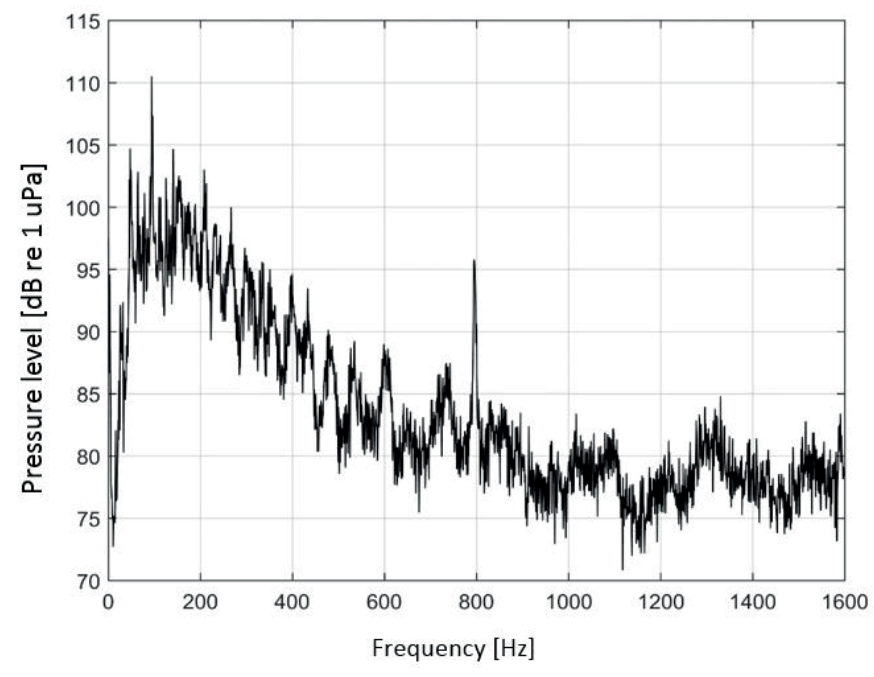

Fig. 1. Narrow-band spectrum recorded on a cargo ship sailing in the Gulf of Gdansk.

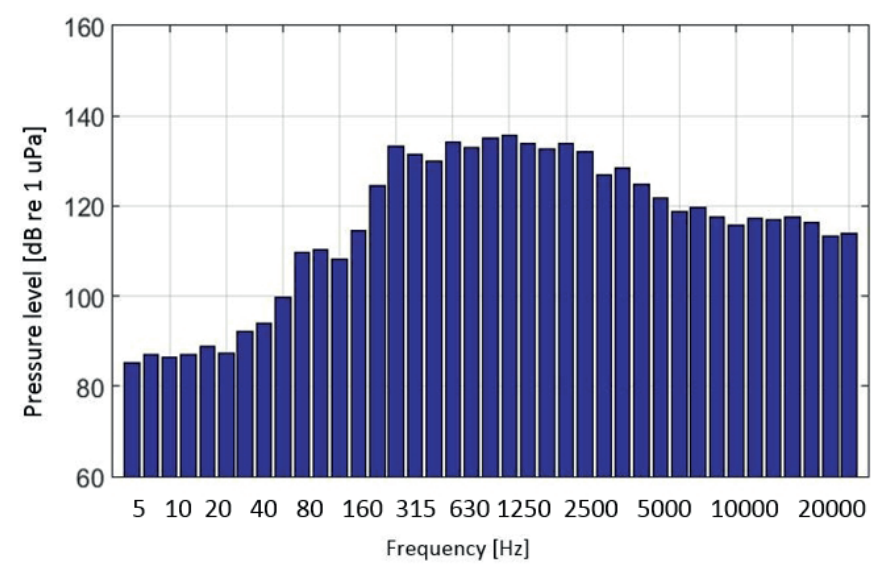

Fig. 2. 1/3 octave spectrum recorded on a cargo ship sailing in the Gulf of Gdansk, 


\section{THEORY - IDEAL WAVEGUIDE}

The analysis of the acoustic field distribution in shallow sea is a complex problem, difficult to put into the framework of a mathematical model. By replacing the shallow sea with a flat acoustic system and assuming that the boundary surfaces of the half spaces limiting the water layer are parallel to each other, we will be able to determine the acoustic field distribution in such a layer.

It is necessary to bear in mind physical simplifications introduced by this model of shallow sea. Usually, the free surface is a stochastically determined surface, and not a plane. The bottom surface is also determined randomly. Solutions for special cases of this problem can be found in ${ }^{5,14-16}$.

The problem of propagation of acoustic disturbances in a medium is usually solved using methods of either wave acoustics or geometrical acoustics. Both approaches allow the user to solve the problem of acoustic disturbance propagation in the water layer, and lead to the same formula describing the acoustic velocity potential.

Assuming that the density and the speed of sound characterizing the medium are constant, we obtain the following formula

$$
\varphi(R)=\frac{2 \pi i}{h} e^{i \frac{\pi}{4}} \sum_{n=0}^{\infty} \cos \frac{\pi}{h}\left(n+\frac{1}{2}\right) z_{0} \cos \frac{\pi}{h}\left(n+\frac{1}{2}\right) z \sqrt{\frac{2}{\pi k_{n} r}} e^{k_{n} r}
$$

where

$$
k_{r n}=k \sqrt{1-\left[\frac{c}{\omega} \frac{\pi}{h}\left(n+\frac{1}{2}\right)\right]^{2}},
$$

$\omega=2 \pi f$ is the angular frequency, $h$ denotes the depth of the water layer, $\mathrm{k}$ is the wave number, $z_{0}$ is the depth of the zeroorder sound source, $n$ is the mode number, and $z$ and $r$ are the vertical and horizontal coordinate, respectively.

Formula (1) represents a series of wave modes that propagate along the layer with different group velocity defined as

$$
u_{n}=\frac{d \omega}{d k_{r n}}=c \sqrt{1-\left[\frac{c}{\omega} \frac{\pi}{h}\left(n+\frac{1}{2}\right)\right]^{2}} .
$$

The $n$-th wave mode will be able to propagate in a layer with depth $h$ without strong attenuation, provided that the following criterion is met

$$
k_{r n} \geq 0
$$

Condition (4) induces the limit for length $\lambda$ of waves that can propagate in the layer $\mathrm{h}$

$$
\lambda \leq 4 h
$$

with boundary conditions determined as

$$
\begin{array}{ll}
\left.\frac{\partial p(r, z)}{\partial z}\right|_{z=0}=0 & \\
\left.p(r, z)\right|_{z=h}=0 & \text { for } 0<r<\infty
\end{array}
$$

The layer constitutes an acoustic waveguide in which acoustic perturbations propagate in the form of wave modes. Such a waveguide is a classic example of dispersion medium of the second kind ${ }^{6}$.

\section{PROPAGATION OF ACOUSTIC WAVES IN THE WATER LAYER OVER A BOTTOM WITH FINITE ACOUSTIC IMPEDANCE}

The case of propagation of acoustic waves generated by a source of zero order, discussed in the previous chapter, corresponded to idealized conditions. The introduced simplifications concerned, in particular, the lower half space. In reality, the sea bottom with properties close to these of the perfectly rigid medium is rather an exception ${ }^{17,18,19}$. At the same time the assumption allowing us to simplify the effects occurring on the water-air boundary is, as a rule, always valid, as the acoustic impedance of water is about 3500 times as high as that of air ${ }^{13,14}$.

On the lower boundary of the system, the situation is different, as the acoustic impedance of the sea bottom is typically close to that of water. In the case of sandy seabed, the acoustic impedance of water is only four times as low as the impedance of the sea bottom. For silty and clayey bottom this ratio is even lower. Therefore, some portion of acoustic energy will be transferred from the water layer to the bottom, which will result in the decrease of the acoustic pressure level with the increasing distance. In the following section we will derive theoretical relationships that will lead to an acoustic pressure drop pattern different than this obtained from the formula.

After some transformations, given in detail in work ${ }^{2}$, we finally obtain,

$$
\varphi(R)=\frac{2 \pi i}{h} \sum_{n=0}^{\infty} \cos \frac{\pi}{h}\left(n+\frac{1}{2}\right) z_{0} \cos \frac{\pi}{h}\left(n+\frac{1}{2}\right) z e^{i \frac{\pi}{4}} \sqrt{\frac{2}{\pi k_{r n} r}} e^{i k_{r m} r} e^{-\delta_{2 n} r},
$$

where

$$
\delta_{2 n}=\frac{-\pi\left(n+\frac{1}{2}\right)}{h} \frac{\ln \left|\hat{b}_{1}\left(\alpha_{n}^{0}\right)\right|}{2 \sqrt{(h k)^{2}-\left[\pi\left(n+\frac{1}{2}\right)\right]^{2}}},
$$

$$
\hat{b}_{1}(\theta)=\frac{m \cos \theta-\sqrt{n_{1}^{2}-\sin ^{2} \theta}}{m \cos \theta+\sqrt{n_{1}^{2}-\sin ^{2} \theta}},
$$




$$
\alpha_{n}^{0}=\arcsin \frac{\pi\left(n+\frac{1}{2}\right)}{k h} .
$$

The impact of sea bottom properties on acoustic wave propagation is included in the expression $\exp \left(-\delta_{2 \mathrm{n}} \mathrm{r}\right)$. When deriving expression (7), the modulus of the reflection coefficient constituting the multiplier at the hyperbolic functions was assumed equal to one. This allowed us to obtain a simple representation of wave modes propagating along the depth of the layer. However, the actual value of the reflection coefficient responsible for attenuation of individual wave modes was retained in the exponent.

Relationship (7) describes the acoustic field generated by a zero-order point source. Comparing it with (1) we can see that it includes additional terms, which represent acoustic potential decrease with the increasing distance. This decrease is larger than that provided by formula (1).

The above derived relationships, and in particular the results to which they lead, will be further used in numerical investigations of the impact of sea bottom properties on the acoustic field, and confronted with the results measured in the experiment. The reflection coefficients for different sea bottom materials have been determined by the authors by means of the impulse method.

\section{NUMERICAL INVESTIGATIONS}

The influence of acoustical parameters of the bottom on sound field distribution was examined for the following conditions:

- Depth of sea, $\mathrm{h}=10 \mathrm{~m}$,

- Sound speed in water, $c=1500 \mathrm{~m} / \mathrm{s}$,

- Density of water, $\rho=1000 \mathrm{~kg} / \mathrm{m}^{3}$,

- Depth of sound source, $\mathrm{z} 0=1 \mathrm{~m}$.

The analysed low and mid frequencies ranged from $125 \mathrm{~Hz}$ to $1500 \mathrm{~Hz}$.

Four kinds of bottom were considered:

- Ideal bottom without attenuation;

- Medium silt: $\mathrm{m}=\rho_{1} / \rho=1.147 ; \mathrm{n}=\mathrm{c}_{1} / \mathrm{c}=0.9801$;

- Fine sand: $\mathrm{m}=\rho_{1} / \rho=1.2236 ; \mathrm{n}=\mathrm{c}_{1} / \mathrm{c}=1.0364$;

- Fine gravel: $\mathrm{m}=\rho_{1} / \rho=2.4923 ; \mathrm{n}=\mathrm{c}_{1} / \mathrm{c}=1.338$.

Consecutive sets of figures allow us to assess how the acoustic waves propagate over different types of bottom. As the first step, we consider the propagation of a low-frequency wave, $f=125 \mathrm{~Hz}$, over different types of bottom sediments.

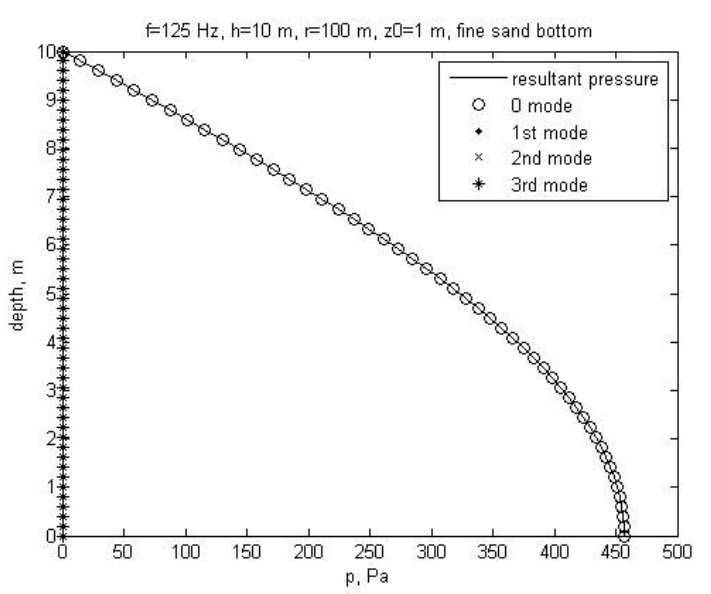

Fig. 3. Modes at the distance of $100 \mathrm{~m}$ over fine sand bottom; $f=125 \mathrm{~Hz}, \lambda=12 \mathrm{~m}$

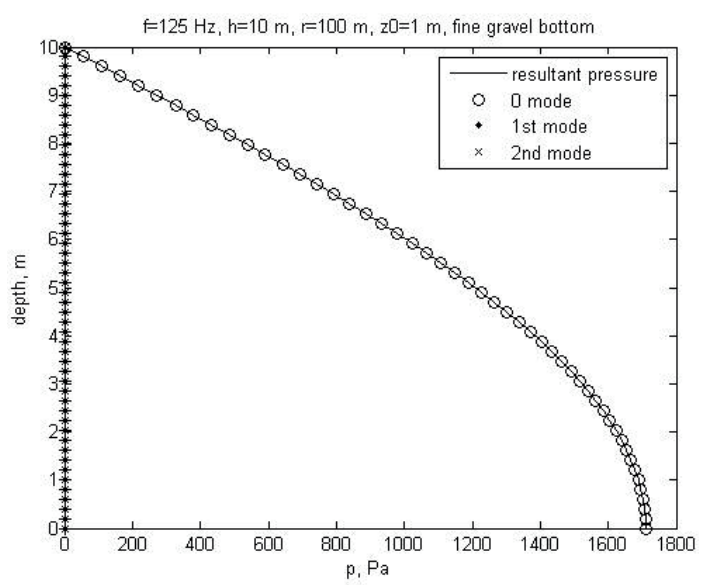

Fig. 4. Modes at the distance of $100 \mathrm{~m}$ over fine gravel bottom; $f=125 \mathrm{~Hz}, \lambda=12 \mathrm{~m}$

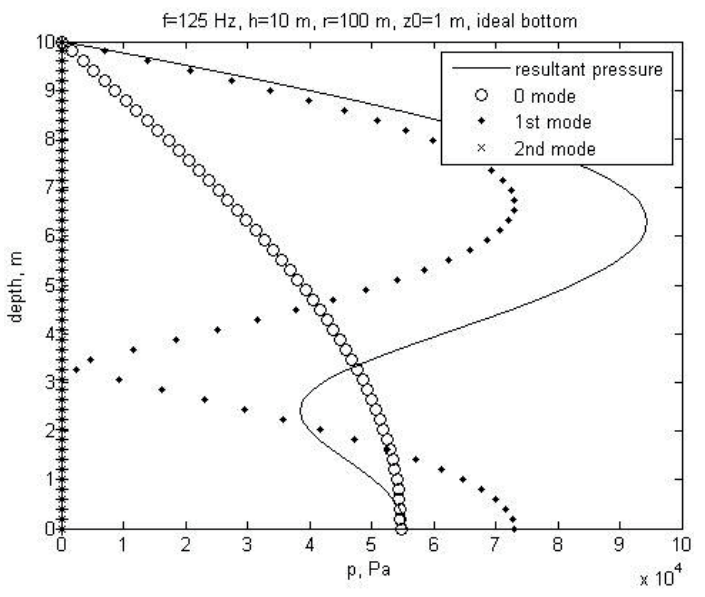

Fig. 5. Modes at the distance of $100 \mathrm{~m}$ over ideal bottom; $f=125 \mathrm{~Hz}, \lambda=12 \mathrm{~m}$

The $125 \mathrm{~Hz}$ wave is attenuated at a very short distance from the source. Independently of the type of bottom, only the 0 mode is created.

The second series of figures illustrate the propagation of modes when the $400 \mathrm{~Hz}$ wave is radiated. 


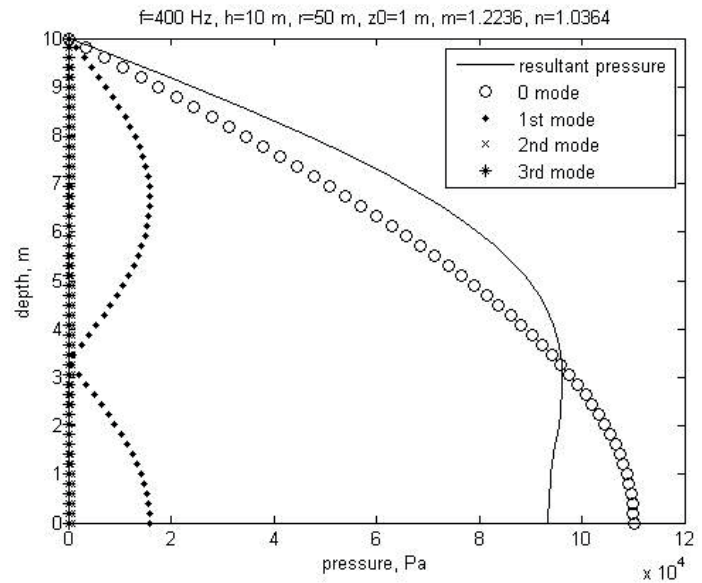

Fig. 6. Modes at the distance of $50 \mathrm{~m}$ over fine sand bottom; $f=400 \mathrm{~Hz}, \lambda=3.75 \mathrm{~m}$

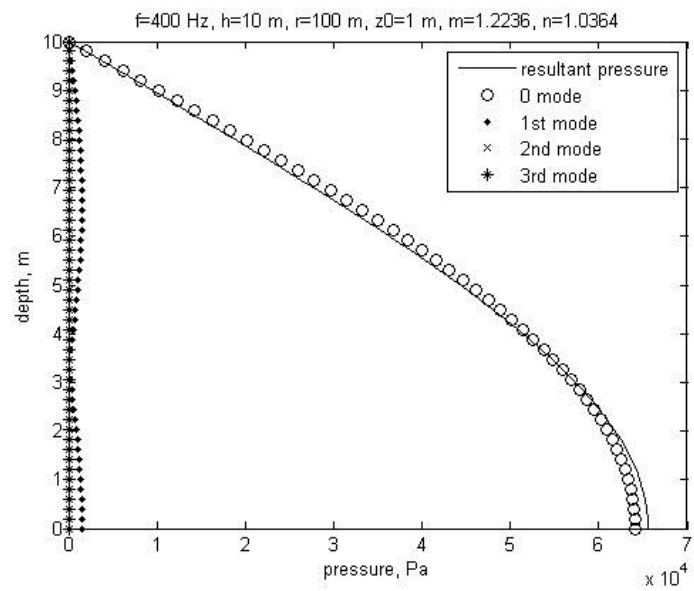

Fig. 7. Modes at the distance of $100 \mathrm{~m}$ over fine sand bottom; $f=400 \mathrm{~Hz}$, $\lambda=3.75 \mathrm{~m}$

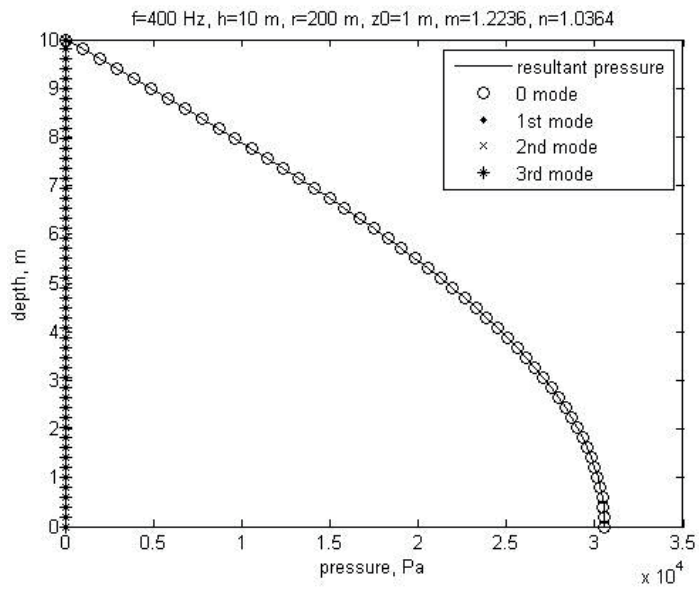

Fig. 8. Modes at the distance of $200 \mathrm{~m}$ over fine sand bottom; $f=400 \mathrm{~Hz}$, $\lambda=3.75 \mathrm{~m}$

In this case, higher modes occur in the vicinity of the source, but they are strongly attenuated. Consequently, only the 0 mode propagates at the distance of $200 \mathrm{~m}$ from the source.
The next considered wave frequency was $750 \mathrm{~Hz}$. The following set of figures shows changes in mode composition in the 10-m deep water layer, at different distances from the source over the fine sand bottom.

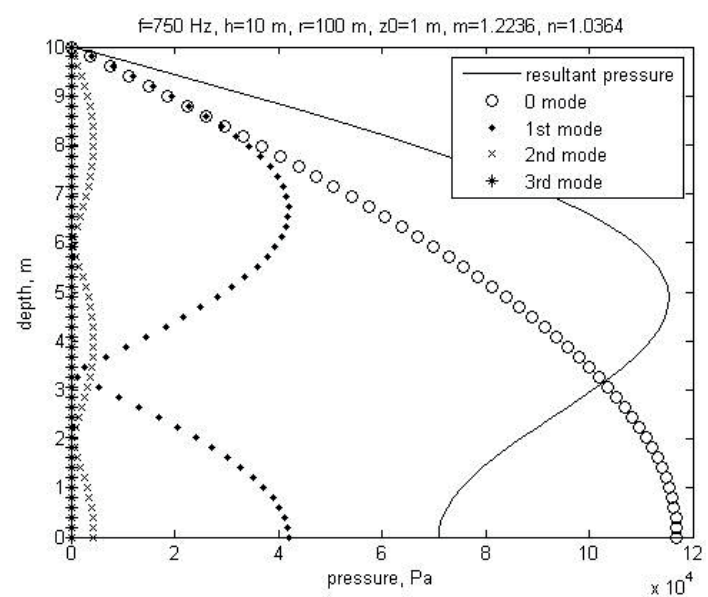

Fig. 9. Modes at the distance of $100 \mathrm{~m}$ over fine sand bottom; $f=750 \mathrm{~Hz}, \lambda=2 \mathrm{~m}$

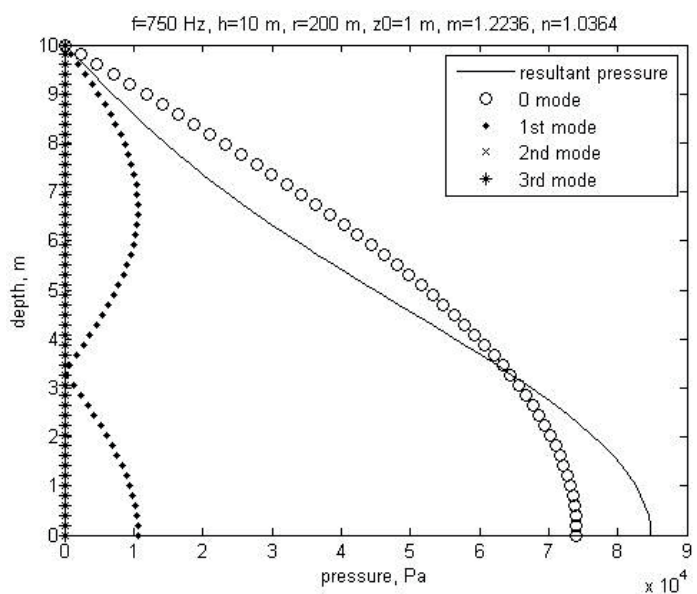

Fig. 10. Modes at the distance of $200 \mathrm{~m}$ over fine sand bottom; $f=750 \mathrm{~Hz}, \lambda=2 \mathrm{~m}$

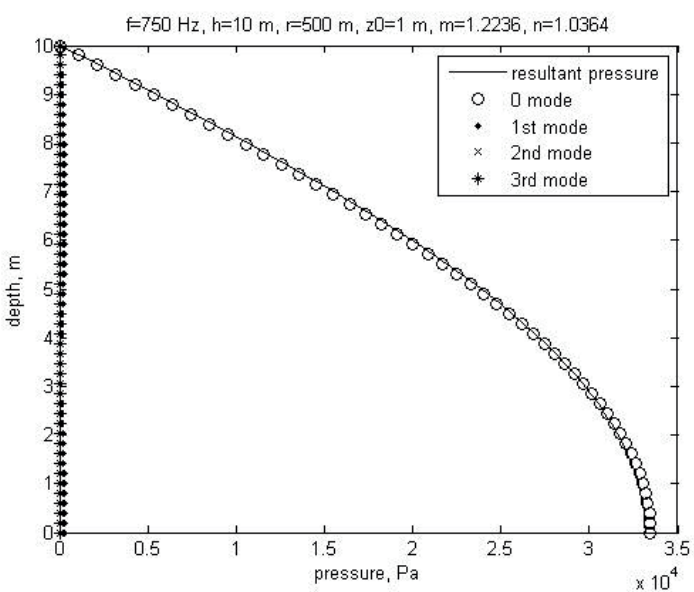

Fig. 11. Modes at the distance of $500 \mathrm{~m}$ over fine sand bottom; $f=750 \mathrm{~Hz}, \lambda=2 \mathrm{~m}$ 
Higher-frequency waves propagate in modes formed over longer distances. When the $750 \mathrm{~Hz}$ wave is radiated in the given conditions, the presence of mode is noticeable at the distance of $500 \mathrm{~m}$ from the source.

The next set of data allows us to assess the influence of bottom properties on modal propagation. Figs. 12-15 show modes of the $1500 \mathrm{~Hz}$ wave at the distance of $1000 \mathrm{~m}$, when it propagates over silty, sandy, or gravelly bottom, and over an ideal non-attenuating bottom.

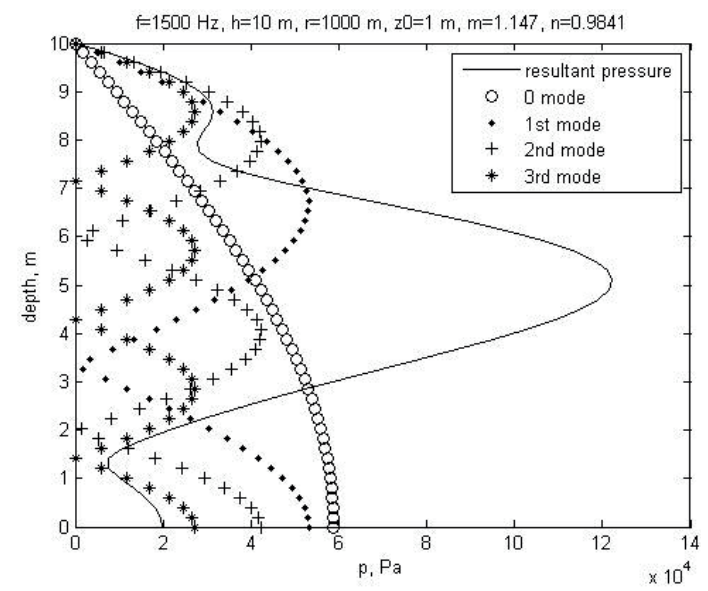

Fig. 12. Modes at the distance of $1000 \mathrm{~m}$ over medium silt bottom; $f=1500 \mathrm{~Hz}$, $\lambda=1 \mathrm{~m}$

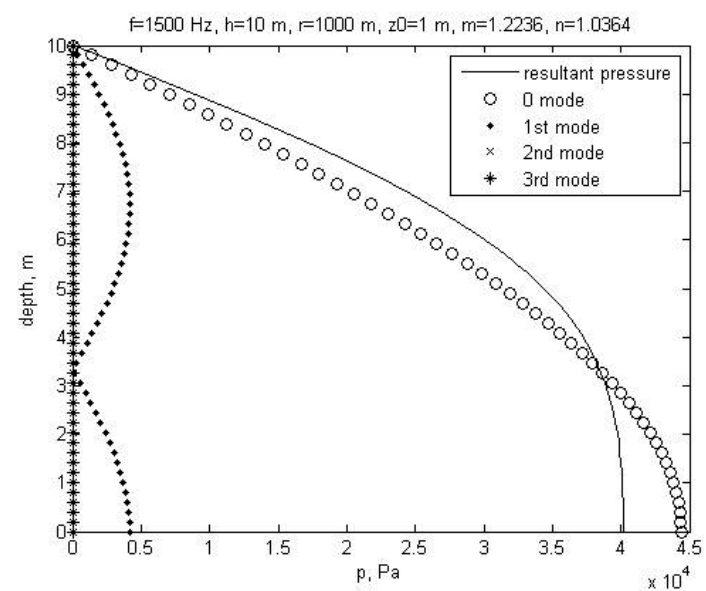

Fig. 13. Modes at the distance of $1000 \mathrm{~m}$ over fine sand bottom; $f=1500 \mathrm{~Hz}, \lambda=1 \mathrm{~m}$

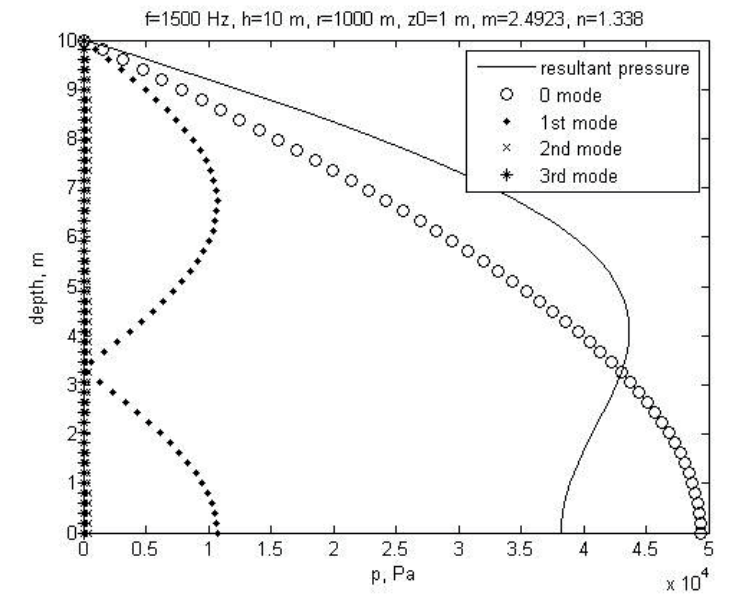

Fig. 14. Modes at the distance of $1000 \mathrm{~m}$ over fine gravel bottom; $f=1500 \mathrm{~Hz}, \lambda=1 \mathrm{~m}$

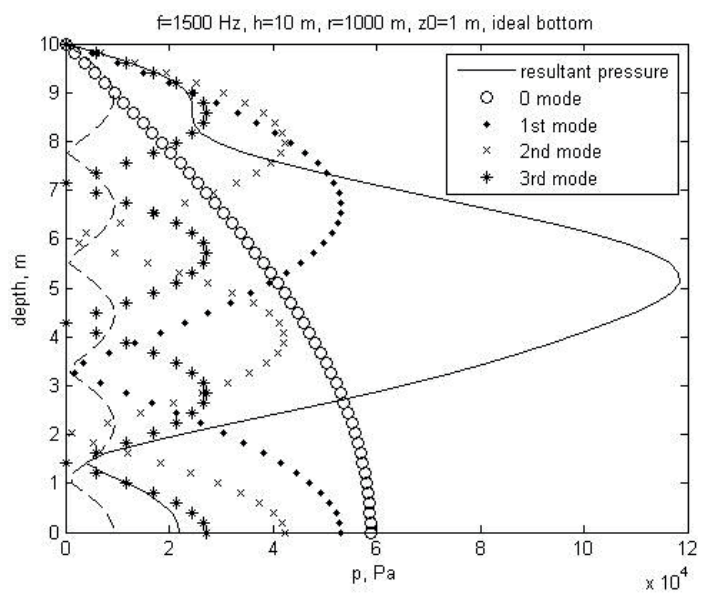

Fig. 15. Modes at the distance of $1000 \mathrm{~m}$ over ideal bottom; $f=500 \mathrm{~Hz}, \lambda=1 \mathrm{~m}$

The set of modes for the case of "soft" bottom, with acoustic parameters similar to those of water, is large, nearly the same as for the ideal waveguide.

The following pictures show how the resultant pressure changes with the increasing distance, for $1500 \mathrm{~Hz}$ wave propagation over different type of bottom sediments.

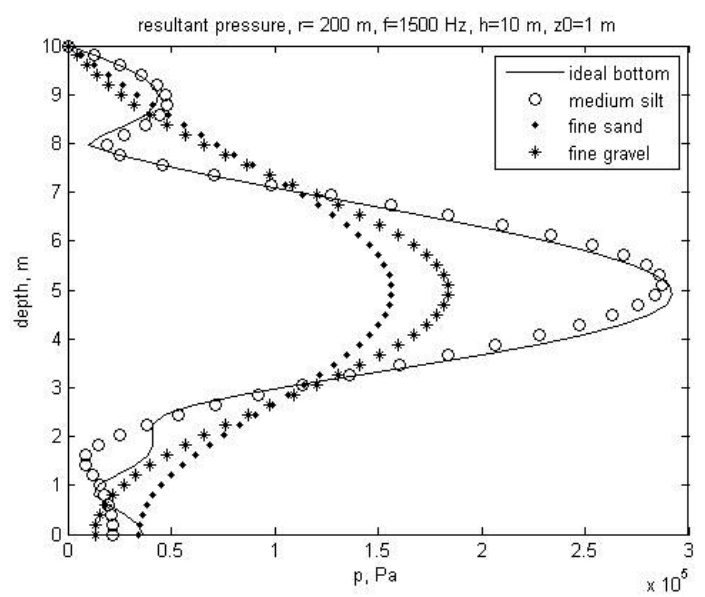

Fig. 16. Resultant pressure at the distance of $200 \mathrm{~m}$ over different types of bottom; $f=1500 \mathrm{~Hz}, \lambda=1 \mathrm{~m}$ 


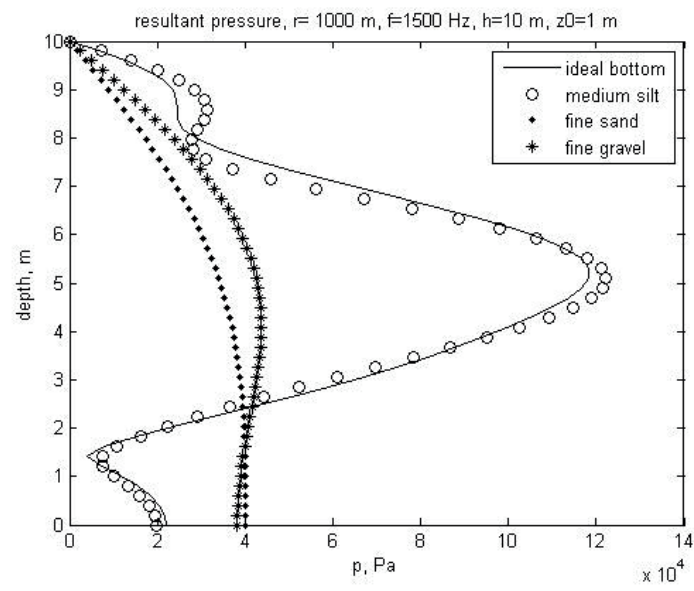

Fig. 17. Resultant pressure at the distance of $1000 \mathrm{~m}$ over different types of bottom; $f=1500 \mathrm{~Hz}, \lambda=1 \mathrm{~m}$

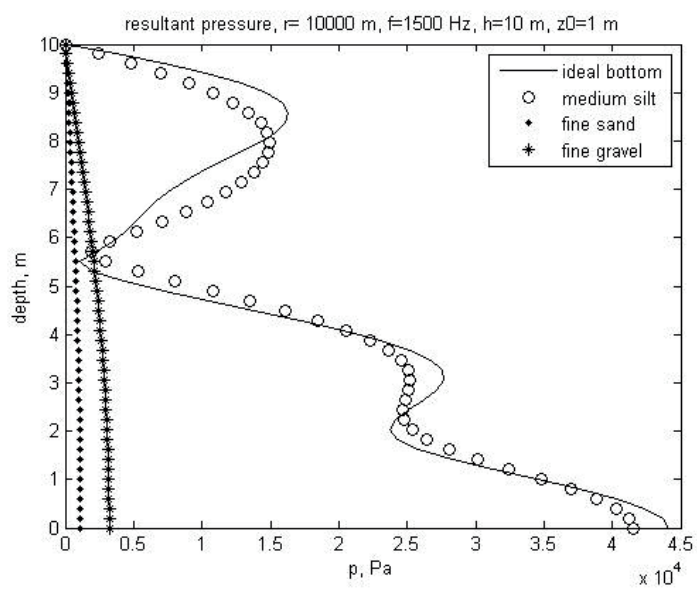

Fig. 18. Resultant pressure at the distance of $10000 \mathrm{~m}$ over different types of bottom; $f=1500 \mathrm{~Hz}, \lambda=1 \mathrm{~m}$

The presence of higher modes and their influence on the resultant pressure is visible up to the distance of about $1000 \mathrm{~m}$ from the source for sandy and gravelly bottom. Then the mode 0 is dominating. At the same time for silty bottom, higher modes propagate over very long distances with small attenuation.

\section{CONCLUSIONS}

The propagation of acoustic waves in a flat-parallel water layer with the determined boundary conditions and the velocity of sound assumed constant in the layer, occurs in the form of wave modes that move with certain group velocity.

The ultimate theoretical result derived in this paper offers the possibility to estimate transmission losses for certain idealized cases, i.e. where both the free sea surface and the bottom can be considered planes. It can be noticed that the water layer reveals discriminative properties that prevent propagation of waves with length exceeding $\lambda=4 h$ for the conditions adopted in this study. As a result of the assumption that $c=$ const, the acoustic pressure distribution ignores the phenomenon of acoustic refraction.

The presented results point to the important influence of sea bottom properties on modal acoustic wave propagation. The knowledge of the vertical pressure distribution combined with the presented theory can be helpful in determining the type of bottom sediments.

\section{ACKNOWLEDGMENTS}

The research was partially supported by the National Centre for Research and Development, Grant No DOBR/0020/R/ ID3/2013/03 and the Ministry for Sciences and Higher Education, under the scheme of Funds for Statutory Activity of Gdansk University of Technology and Polish Naval Academy.

\section{REFERENCES}

1. W. A. Kuperman and J. F. Lynch, Shallow-Water Acoustics, Physics Today, October (2004) 55-61.,

2. E. Kozaczka, The Propagation of the Acoustic Disturbances in the Shallow Water, in Hydroacoustics of Shallow Water, eds. E. Kozaczka and G. Grelowska, (Institute of Fundamental Technological Research Polish Academy of Sciences, Warszawa, 2013), pp. 31-52.

3. L. Guieysse and P. Sabathe, Acoustique sous-marine, Deuxième édition, (Paris, 1964).

4. E. Kozaczka and G. Grelowska, Shipping low frequency noise and its propagation in shallow water, Acta Physica Polonica A, 119, 6A (2011) 1009-1012.

5. I. Tolstoy and C. S. Clay, Ocean Acoustics (Mc Graw-Hill Company, New York, 1966).

6. K. F. Herzfeld and T. A. Litovitz, Absorption and Dispersion of Ultrasonic (New York,1965).

7. M. F. McKenna, D. Ross, S. M. Wiggins and J. A. Hildebrand, Underwater radiated noise from modern commercial ships, J. Acoust. Soc. Am. 131, 1 (2012) 92-103.

8. D. Ross, Ship sources of ambient noise, IEEE Journal of Oceanic Engineering, 30 (2005), 257-261.

9. P. T. Arveson and D. T. Vendittis, Radiated noise characteristics of modern cargo ship, J. Acoust. Soc. Am., 107, 1 (2000) 118-129.

10. E. Kozaczka, J. Domagalski, G. Grelowska and I. Gloza, Identification of hydroacoustic waves emitted from 
floating units during mooring tests, Polish Maritime Research, 14, 4 (2007) 40-12.

11. D. Ross, Mechanics of Underwater Noise (Pergamon, New York, 1976).

12. R. J. Urick, Principles of Underwater Sound, Chap. 10, (Mc Graw-Hill, New York, 1975).

13. G. Grelowska, E. Kozaczka, S. Kozaczka and W. Szymczak, Underwater noise generated by a small ship in the shallow sea, Archives on Acoustics, 38, 3 (2013) 351-356.

14. I. Malecki I., Physical foundations of technical acoustics, (Pergamon Press, 1969).

15. L. M. Briechowskich, Waves in layered media, 2nd Edition (Academic Press, New York 1980).

16. E. L. Shenderov, Wave problems in hydroacoustics, (Sudostroenie Press, Leningrad, 1972).

17. G. Grelowska and E. Kozaczka, Sounding of layered marine bottom - modeling investigations, Acta Physica Polonica A, 118, 1 (2010) 66-70.

18. E. Kozaczka, G. Grelowska and S. Kozaczka, Images of the seabed of the Gulf of Gdansk obtained by means of the parametric sonar, Acta Physica Polonica A, 118, 1 (2010) 91-94.

19. E. Kozaczka, G. Grelowska, W. Szymczak and S. Kozaczka, The examination of the upper layer of the seabed using acoustic methods, Acta Physica Polonica A, 119, 6A (2011) 1091-1094.

\section{CONTACT WITH THE AUTHOR}

Eugeniusz Kozaczka

e-mail:eugeniusz.kozaczka@wp.pl

Gdansk University of Technology Faculty of Ocean Engineering and Ship Technology

Narutowicza 11/12

80-233 Gdansk

Poland 